\title{
Original
}

\section{Estrés laboral del Profesional de Enfermería en Unidades Críticas}

\author{
Work-Related Stress in Nurses at Critical Care Units \\ Pía Mabel Molina-Chailán',2, Marisol Muñoz-Coloma ${ }^{1,3}$, Giannina Schlegel-San Martín ${ }^{1,2}$ \\ 1. UCl médico-quirúrgica Hospital Guillermo Grant Benavente Concepción-Chile. \\ 2. Facultad de Enfermería Universidad de Concepción-Chile. \\ 3. Universidad San Sebastián Concepción-Chile.
}

Recibido: 06-07-2019

Aceptado: 13-08-2019

\section{Correspondencia}

Pía Mabel Molina Chailán

Dirección San Martin 1436

Concepción-Chile. Chile

Correo electrónico: piamabel@gmail.com

Resumen

Introducción: El cuidado de las personas expone a los trabajadores a estrés, que puede estar relacionado con carga laboral, ambiente psicológico y social, donde las altas exigencias del trabajo y la oportunidad de controlarlas son escasas.

Objetivo: Identificar los estresores laborales que generan riesgos en la salud del equipo de enfermería de las Unidades de Cuidados Intensivos.

Método: Estudio descriptivo transversal, muestra por conveniencia de 37 enfermeros, se utilizó Nurses Stress Scale de Gray-Toft y Anderson, midiendo 7 factores desencadenantes de estrés en el trabajo distribuidos en 3 grupos: carga laboral, ambiente psicológico y ambiente social.

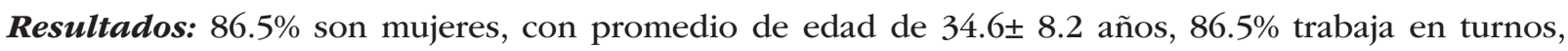
antigüedad trabajo $8.9 \pm 7.6$ años. El 48.6\% de los estresores están relacionados con carga laboral, dado por la realización de tareas que no corresponden a enfermería y la falta de tiempo para dar apoyo emocional a los pacientes. El ambiente psicológico el factor estresor es la realización de procedimientos que resultan dolorosos para el paciente, mientras que el ambiente social, el 70.2\% el factor estresor es la ausencia del profesional médico cuando el paciente está muriendo.

Conclusiones: los profesionales de enfermería son mayoritariamente mujeres, con varios años de experiencia laboral, donde el principal factor desencadenante de estrés es la sobrecarga laboral lo que conlleva a una pérdida de control del cuidado de enfermería. El sufrimiento y dolor del paciente son factores que generan estrés psicológico, mientras los estresores del entorno social están menos presentes.

Med Segur Trab (Internet). 2019;65(256):177-185

Palabras Claves (DeCS): estrés laboral; enfermería; carga de trabajo; cuidados críticos.

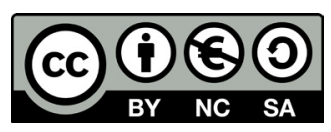

https://creativecommons.org/licenses/by-nc-sa/4.0/ 


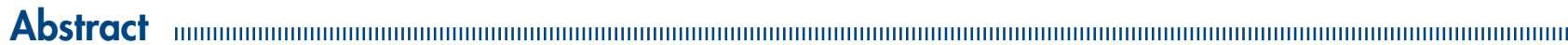

Background: The care of people exposes workers to stress, which may be related to workload, psychological and social environment, where the high demands of working life and the opportunity to control them are scarce. Units.

Objective: To identify the occupational stressors generating risks in nursing health at Intensive Care

Method: Cross-sectional descriptive study, convenience sample $n=37$ nurses. The Nursing Stress Scale by Gray-Toft and Anderson was used, measuring 7 stress factors at work distributed in 3 groups: workload, psychological environment and social environment.

Results: $86,5 \%$ was women, with an average age of $34,6 \pm 8.2$ years, $86,5 \%$ work in shifts, seniority work $8,9 \pm 7.6$ years. $48,6 \%$ of the stressors are related to workload, given by the task performance that do not correspond to nursing job and the lack of time to provide emotional support to patients. In the psychological environment the stressor was the performance of procedures that are painful for the patient, while in the social environment, $70,2 \%$ the stressor was the absence of professional staff needed for the dying patient.

Conclusions: Nursing professionals are mostly women, with several years of work experience, the workload being the main triggering stress, leading to a loss of control over nursing care. Although the stressors of the social environment are less present, the suffering and pain of the patient are psychological stress-generating factors.

Med Segur Trab (Internet). 2019;65(256):177-185

Keywords (DeCS): occupational stress; nursing; workload; critical care. 


\section{INTRODUCCIÓN}

El cuidado de las personas expone a los trabajadores a estrés y riesgos psicosociales que en ocasiones puede llevar a la enfermedad laboral ${ }^{1}$. El fisiólogo Hans Seyle, en 1946, a través de su estudio del Síndrome de Adaptación General, definió estrés como una respuesta no específica del cuerpo frente a cualquier demanda, de forma que si su duración es suficientemente prolongada el organismo puede entrar en una etapa de agotamiento $^{2}$. De esta manera, la tensión psíquica y física que aparece en respuesta a las demandas del medio hospitalario puede tener manifestaciones cognitivas, emocionales, conductuales y fisiológicas. La Organización Mundial de la Salud (OMS) señala que estrés laboral es "un patrón de reacciones psicológicas, cognitivas y conductuales que se dan cuando los trabajadores se enfrentan a exigencias ocupacionales que no corresponden con su nivel de conocimiento, destreza o habilidades". Por lo tanto, cada profesión tiene sus propias causas de estrés ${ }^{2,3}$.

Para poder explicar las situaciones de estrés desarrolladas en el trabajo, Robert Karasek, en 1979, diseña un modelo de evaluación del estrés laboral denominado Modelo Demanda-Control, el cual establece que este tipo de estrés es resultante del desequilibrio entre demandas psicológicas y control sobre el trabajo, pudiendo ocasionar consecuencias nocivas a la salud de los trabajadores. Señala que no sólo las demandas psicológicas que el trabajo impone definen la experiencia del estrés, sino también el grado de control que los trabajadores tienen en su tarea, lo que funciona como un mecanismo moderador de tales demandas. De esta forma, el estrés laboral surgiría en aquellas actividades cuyas exigencias del trabajo son elevadas y la oportunidad de control es escasa ${ }^{4,5}$.

El modelo propuesto por Karasek puede explicar 4 situaciones de trabajo específicas que sugieren riesgos diferenciados a la salud (Figura 1): el cuadrante $n^{\circ} 1$ : alta exigencia (alta demanda psicológica y de bajo control) es la situación más riesgosa; el cuadrante $\mathrm{n}^{\circ} 3$ se establece como de baja exigencia y menos riesgosa (baja demanda y alto control); mientras el cuadrante $\mathrm{n}^{\circ} 2$ : trabajo activo (alta demanda y alto control categoría de menor riesgo) situación de estrés positivo moderado y cuadrante $\mathrm{n}^{\circ} 4$ : trabajo pasivo (baja demanda y bajo control) situación de estrés moderada. La interacción entre altas demandas psicológicas del trabajo y bajo control sobre las actividades configuradas con una situación de alta exigencia desencadenando estrés laboral ${ }^{6}$.

Figura 1. Modelo Demanda- Control de Robert Karasek, 1979.

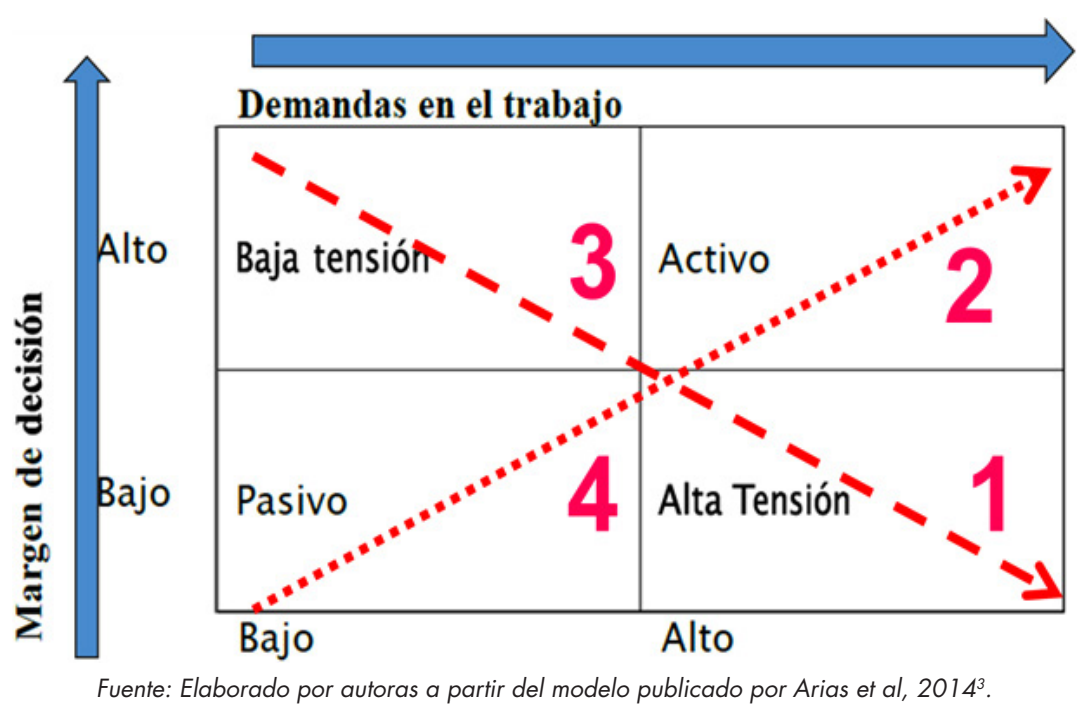

El consejo internacional de enfermeras (CIE) señala que la presencia de estrés no siempre es de origen asistencial, sino que existen otros aspectos laborales que pueden ser perjudiciales y están relacionados con características del sistema de salud y su 
organización. Un desajuste entre el trabajador, el puesto de trabajo y la propia institución, producen una infravaloración e insatisfacción personal del profesional ante el trabajo que desarrolla, es así, como los profesionales de enfermería deben asumir la responsabilidad de identificar problemas y cuestiones que se plantean en su entorno laboral y ofrecer soluciones al respecto ${ }^{5,7}$

Algunos estudios han identificado diversos factores de estrés laboral en profesionales de enfermería. En Colombia, Castillo et al $^{8}$, en 2014, utilizando el mismo instrumento en enfermeras y enfermeros de los servicios de urgencia, hospitalización, cirugía y consulta externa, se observó presencia de estrés asociada a factores personales y laborales, vinculado a los servicios en que se trabaja y al tipo de contratación. En Chile en 2015, similar estudio realizado por Muñoz et $\mathrm{al}^{9}$, demostró que el $48.5 \%$ de las enfermeras generaron algún grado de estrés, principalmente relacionado con el ambiente psicológico y social, manifestado al observar el sufrimiento del paciente; realizar procedimientos que suponen experiencias dolorosas al usuario; tener que realizar tareas no relacionadas con enfermería e insuficiente personal para cubrir adecuadamente el trabajo de la unidad. En cambio, el estudio de Carrillo-García et al $^{10}$, en España 2018, describió que el estrés laboral se manifestó en un nivel moderado principalmente relacionado al déficit de apoyo social por parte de los superiores.

De acuerdo con la evidencia empírica, a la importancia del fenómeno para enfermería y el compromiso con la salud laboral, se ha propuesto como objetivo identificar los estresores laborales que generan riesgos en la salud del equipo de enfermería de las Unidades de Cuidados Intensivos Pediátricos y Adultos de un Hospital Público en Chile.

\section{MÉTODOS}

Estudio descriptivo transversal, universo compuesto por 45 profesionales de enfermería de dos unidades de cuidados intensivos (pediátrica y adulto) de la ciudad de Concepción, Chile. La selección de los participantes fue por conveniencia, 37 enfermeros autorizaron participar representando el 82.2\% del universo, logrando un número pequeño, pero suficiente de sujetos para analizar las condiciones de estrés laboral en la unidad. Entre los criterios de inclusión se consideró:1) profesional de enfermería titular o contratado perteneciente a las unidades de cuidados intensivos seleccionadas; 2) profesional de enfermería reemplazante con más de 6 meses en la unidad. Los criterios de exclusión fueron: 1) alumnos de enfermería; 2) rechazo a participar en el estudio 3) profesionales con licencias médicas, feriados legales y/o descansos compensatorios durante el periodo de estudio. Según estos criterios, 4 profesionales negaron participar, 3 se encontraban con feriado legal y 1 profesional llevaba menos de 6 meses en la unidad. El instrumento recolector de la información es autoadministrado y consistió en dos cuestionarios: 1) Cuestionario de antecedentes sociodemográficos; 2) Escala de Estrés en Enfermería de Gray-Toft y Anderson.

a) Cuestionario Sociodemográfico: Cuestionario tipo Likert, confeccionado por las investigadoras, que consta de nueve preguntas que describen la situación sociodemográfica de los encuestados. Seis de ellas con respuesta dicotómica y tres con respuesta abierta.

b) Escala de Estresores en Enfermería (Nursing Stress Scale, NSS), creada por GrayToft \& Anderson en su versión original, traducida y validada al español por Pons \& Escribá en $1998^{11}$. Consta de 34 ítems, con formato de respuesta tipo Likert "nunca", "alguna vez", "frecuentemente" y "muy frecuentemente". El rango de puntuaciones es de 0 a 102, de modo que, a mayor puntuación, mayor nivel de estrés laboral. estos ítems están divididos en tres grupos, los que a su vez se subdividen, estableciendo finalmente 7 factores importantes en el desarrollo del estrés en enfermería:

Grupo 1: Factor 1: Carga de trabajo. 
Grupo 2: Ambiente Psicológico.

Factor 2: muerte y sufrimiento.

Factor 3: preparación insuficiente.

Factor 4: falta de apoyo.

Factor 5: incertidumbre en el tratamiento.

Grupo 3: Ambiente Social.

Factor 6: en el hospital: problema con los médicos.

Factor 7: problemas con otros miembros del equipo de enfermería.

Con el objetivo de conocer el comportamiento del instrumento, se realizó una prueba piloto a 10 enfermeros de diversas unidades de cuidados intensivos de la región, esta prueba permitió establecer 10 a 15 minutos el tiempo aproximado de respuesta del instrumento, no detectando problemas en la aplicación de los cuestionarios ni en la comprensión de los ítems, la evaluación de la consistencia interna de la escala dado por el coeficiente Alpha de Cronbach para este estudio fue de 0.87

Considerando los principios éticos propuestos por Ezequiel Emanuel, se solicitó autorización científico- ética de las jefaturas administrativas y clínicas del centro de responsabilidad Paciente Crítico y de las unidades participantes. Posteriormente, a cada profesional encuestado se informó de los objetivos del estudio, su contribución, libertad de participación, aseguramiento del anonimato y la confidencialidad de la información proporcionada, luego se solicitó por escrito la autorización, a través del consentimiento informado.

Los datos fueron obtenidos en el periodo Junio-Julio de 2015. El análisis de la información se realizó a través del paquete estadístico SPSS versión 15 para Windows, se dio respuesta al objetivo de estudio utilizando análisis descriptivo un variado y bivariado, la presentación de las variables se realizó según la naturaleza de estas, el análisis inferencial fue a través de asociación de variables categóricas con un intervalo de confianza 95\% y nivel de significancia $\mathrm{p}<0.05$.

\section{RESULTADOS}

La muestra total de 37 profesionales de enfermería de los cuales el $21.6 \%(n=8)$ corresponden a profesionales de la Unidad de Cuidados Intensivos Pediátricos (UCIP) y el $78.3 \%(n=29)$ a la Unidad Cuidados Intensivos Adultos (UCIA). De acuerdo con el total de enfermeros por unidad los encuestados en la unidad pediátrica equivalen al $61.5 \%$ y al $90 \%$ en la unidad de adultos.

Del total de la muestra, el $86.5 \%(n=32)$ corresponden a mujeres, mientras que el $13.5 \%(n=5)$ son hombres, la edad media fue de $34.6 \pm 8.2$ años con un rango 23 a 56 años. El 73\% $(n=27)$ señala tener pareja estable y el 59.5\% $(n=22)$ tiene hijos.

El $86.5 \%(n=33)$ trabaja en sistema de turnos y el $13.5 \%(n=5)$ en jornada diurna, el $89.2 \%(n=33)$ son contratados, el $5.4 \%(n=2)$ tienen contrato titular y en igual proporción son reemplazantes. Los años de antigüedad de los profesionales es de $8.9 \pm$ 7.6 años con un rango de 6 meses a 32 años, el $27 \%(n=10)$ señala tener un segundo trabajo remunerado.

De un puntaje NSS total de 102 puntos, la media obtenida en la muestra fue de $37.9 \pm 11.6$, el $50 \%$ de los participantes tienen menos de 38 puntos y el 50\% tiene por sobre ese valor, con un rango de 5 a 64 puntos. En la Tabla I, se describen los puntajes obtenidos por cada factor evaluado en NSS. 
Tabla I. Estadísticos descriptivos según puntaje obtenido NSS por factores.

\begin{tabular}{|c|c|c|c|c|}
\hline & Media & DS & Máximo & IC (95\%) \\
\hline Factor 1: Carga de trabajo & 7.00 & 2.84 & 13 & {$[6.05 ; 7.95]$} \\
\hline Factor 2: Muerte y Sufrimiento & 6.46 & 2.09 & 10 & {$[5.76 ; 7.16]$} \\
\hline Factor 3: Preparación insuficiente & 6.11 & 2.66 & 11 & {$[5.22 ; 7.00]$} \\
\hline Factor 4: Falta de apoyo & 3.35 & 1.41 & 7 & {$[2.88 ; 3.82]$} \\
\hline Factor 5: Incertidumbre en el tratamiento & 4.73 & 1.44 & 8 & {$[4.25 ; 5.21]$} \\
\hline Factor 6: Problemas con los médicos & 5.76 & 2.30 & 12 & {$[4.99 ; 6.52]$} \\
\hline Factor 7: Problemas con otros colegas & 4.51 & 2.11 & 8 & {$[3.81 ; 5.22]$} \\
\hline
\end{tabular}

DS: Desviación estándar; Máximo: puntaje máximo obtenido; IC: Intervalo de Confianza 95\%

Los profesionales describen la carga de trabajo (grupo y factor 1) como el principal factor de estrés laboral, el 48.6\% frecuentemente "realiza demasiadas tareas que no son de enfermería" y en similar proporción indican "no tener tiempo suficiente para dar apoyo emocional al paciente".

En el ambiente psicológico (grupo 2), el 43.2\% de los enfermeros/as señalan presentar estrés cuando "ver a un paciente sufrir" (factor 2); el 73\% indica alguna vez haber sentido "miedo a cometer un error en los cuidados de enfermería"; el 70.3\% alguna vez se sintió "insuficientemente preparado para ayudar emocionalmente a la familia" " "... al paciente"; el 62.2\% alguna vez "no pudo manejar bien un equipo especializado". El 54\% afirma que "las interrupciones frecuentes en la realización de sus tareas" son causa de estrés en el factor 4: falta de apoyo. La incertidumbre en el tratamiento (factor 5) indica que el 97.3\% de los profesionales alguna vez o frecuentemente se ha "sentido impotente en el caso de un paciente que no mejora", el $78.4 \%$ alguna vez ha estado en "desacuerdo con el tratamiento de un paciente", mientras que el 27\% afirma que la situación que genera estrés es "tomar la decisión acerca de un paciente cuando el médico no está disponible”.

En el ambiente social, los problemas relacionados con los médicos (factor 6) se observan en las afirmaciones, "el médico no está presente cuando el paciente está muriendo" (70.2\%) y "el médico no está presente en una urgencia médica" (89\%); en los problemas con otros miembros del equipo de enfermería (factor 7) están descritos en las afirmaciones, "problemas con un supervisor" (81.1\%) y "recibir críticas de un supervisor" (86.5\%).

Según la distribución de encuestados por unidad, todos los enfermeros de UCIP afirman muy frecuentemente y frecuentemente "tener interrupciones en la realización de sus tareas". Mientras que el ítem "el médico no está presente cuando un paciente se está muriendo" el $41.3 \%$ responde frecuentemente en la UCIA y el $12.5 \%$ en la UCIP. "Ver a un paciente morir" frecuentemente y muy frecuentemente produce estrés en el 75\% de los enfermero/as UCIP y el 51.7\% en enfermeros/as UCIA. El 24.1\% frecuentemente y muy frecuentemente "recibe críticas de un supervisor" en UCIA, mientras que en UCIP no se registró casos. El ítem "no tener tiempo suficiente para realizar todas mis tareas de enfermería" y "la falta de personal para cubrir adecuadamente el servicio", refieren frecuentemente los enfermeros UCIP en el $75 \%$ y $62.5 \%$, a diferencia de los enfermeros UCIA con $41.4 \%$ y $27.5 \%$ en cada afirmación.

El análisis inferencial de los datos demostró con la prueba Chi-Cuadrado que no existe asociación estadísticamente significativa entre cada factor que componen el instrumento y las variables sociodemográficas de sexo, sistema de turno, religión, tipo de contrato y estado civil.

\section{DISCUSIÓN}

Los profesionales participantes en este estudio en su mayoría son mujeres con una edad promedio de 34.6 años, con hijos y pareja estable sin distinción del estado civil, 
trabaja principalmente en sistema de turnos, y vinculo contractual definido, la antigüedad en la unidad es en promedio de 8.9 años. Este perfil concuerda con las características estudiadas en otros profesionales de enfermería en España, Brasil, Colombia y Chile (1,5,6,8,9,10. $^{\text {. }}$

El puntaje total obtenido por los participantes en promedio fue de 37.9 puntos con una mediana de 38 puntos, el estudio chileno de Muñoz en $2015^{\circ}$, presentó un puntaje corte de 34 puntos correspondiendo al 48.5\% de los encuestados. Los puntajes promedios según los factores estudiados fueron muy similares a los obtenidos por Garza en $2011^{12}$, la media del factor 1: carga de trabajo fue de 7 puntos $\mathrm{v} / \mathrm{s} 6.11$, el factor 2: muerte y sufrimiento con 6.46 puntos v/s 6.71 y el factor 3: preparación insuficiente: $6.1 \mathrm{v} / \mathrm{s} 5.4$ puntos.

Según el análisis por grupos. El grupo 1: carga laboral, presenta proporciones mayores que el resto de los grupos estudiados, considerando que la respuesta más seleccionada por ítem fue "realizar demasiadas tareas que no son de enfermería" y "no tener tiempo suficiente para dar apoyo al paciente”. Castillo en $2014^{8}$, observó proporciones de $51.9 \%$ y $50 \%$ para cada afirmación, a diferencia del estudio chileno de Muñoz en $2015^{\circ}$, solo el $14.4 \%$ indico muy frecuentemente para la primera afirmación. Kogien en $2014^{13}$, señaló que el trabajo de mayor exigencia es un potencial creador de estrés de los enfermeros/as en los servicios de urgencia.

El grupo 2: ambiente psicológico. El 43.2\% de los enfermeros/as indica sentir estrés frecuentemente con la afirmación "ver a un paciente sufrir", en el estudio de Muñoz, $2015^{\circ}$. El $40 \%$ señala frecuentemente sentir estrés por esta afirmación y el $42.9 \%$ en el estudio de Castillo, $2014^{8}$.

El 70.3\% de los encuestados señalo alguna vez "sentirse insuficientemente preparado para ayudar a la familia y paciente", proporciones mayores se observaron en otro estudio?

Los resultados que definen la incertidumbre al tratamiento. Difieren con otras investigaciones, mientras que el $\mathbf{9 7 . 3 \%}$ de los profesionales alguna vez o frecuentemente "se han sentido impotente en el caso que un paciente no mejora" y el $74.4 \%$ alguna vez ha estado "en desacuerdo con el tratamiento de un paciente".

Estudios similares atribuyen como causas de estrés en este factor "la información insuficiente del médico acerca del estado clínico del paciente" (46.7\% Castillo, $\left.2014^{8}\right)$ y el $5.4 \%$ indica frecuentemente "no conocer lo que se puede comunicar a un paciente o su familia sobre su tratamiento" (7.4\%. Muñoz, $2015^{9}$ v/s 52\%. Castillo, 2014 ${ }^{8}$ ).

En el ambiente social, el $70 \%$ de los problemas relacionados con los médicos se describen en la afirmación "el médico no está presente cuando el paciente está muriendo" y el 89\% "el médico no está presente en una urgencia” (54.1\%. Castillo, 2014)

Los problemas con otros enfermeros, el $81.1 \%$ indica alguna vez o frecuentemente "tener problemas con un supervisor", en el estudio de Castillo ${ }^{8}$, el 35.3\% de los encuestados identificó como frecuentemente esta afirmación. El estudio de Carrillo-García, 2018 ${ }^{10}$, también identifica niveles moderados de estrés relacionados con el apoyo recibido por los superiores

El presente estudio no identifico asociación entre las variables sociodemográficas y los factores estudiado con NSS, sin embargo, Rodrígues en $2011^{14}$, señala que los enfermeros/as con prestaciones temporales presentan mayor estrés que los enfermeros/ as con vínculo laboral definitivo. La revisión realizada por Navarro en $2012^{15}$, identifica como una de las causas de estrés laboral el salario y la situación contractual de los profesionales. Mientras que Carrillo-García manifiesta estrés laboral según el apoyo social recibido ${ }^{10}$.

Las limitaciones del estudio se relacionan principalmente con el número de participantes, si bien representan una gran proporción de profesionales de las unidades estudiadas, no es posible generalizar los resultados a la comunidad. 


\section{CONCLUSIONES}

El presente estudio permite identificar las causas que generan estrés en el profesional de enfermería y determinar cuáles son las situaciones que producen determinadas sensaciones y emociones incomodas, dejando a la persona con la decisión de poder enfrentar o evadir dicha actividad.

La sobrecarga laboral es la causa de estrés laboral principal del presente estudio, debido a la pérdida de control de la situación y a la alta demanda asistencial, condicionada por diversas situaciones, tales como, las descritas en la escala NSS.

Las problemáticas laborales relacionadas al trabajo en equipo con otros profesionales se observan en principalmente en la entrega de información y comunicación de los vinculados.

Desde esta perspectiva, es posible que un profesional de enfermería confundido y angustiado entregue cuidados operacionales orientados al tratamiento y no a la persona debido a los factores que producen estrés laboral. Por lo tanto, estrategias de evaluación del trabajo, a través de modelos bien definidos e instrumentos propios de la disciplina, como NSS, permiten gestionar el cuidado de enfermería considerando al cuidador profesional, lo que resulta un desafío para las instituciones de salud, sin dejar de lado la humanización de los cuidados y calidad en la atención.

Se sugiere en las próximas investigaciones considerar variables de estudio como el ausentismo laboral, estímulos laborales, actividades extraprogramáticas y de capacitación.

\section{REFERENCIAS BIBLIOGRÁFICAS}

1. Ansoleaga E. Indicadores de salud mental asociados a riesgo psicosocial laboral en un hospital público. Rev Med Chile [revista en la internet]. 2015 [consultado 16 de octubre de 2018]; 143:47-55. Disponible en: https://scielo.conicyt.cl/pdf/rmc/v143n1/art06.pdf

2. Aguado J, Batíz A, Quintana S. El estrés en personal sanitario hospitalario; estado actual. Med Segur Trab [revista en la internet]. 2013 [consultado 16 octubre de 2018];59(231):259-275. Disponible en: http://scielo. isciii.es/pdf/mesetra/v59n231/revision1.pdf

3. Arias W, Montes I, Masías M. El modelo Demanda Control de Karasek y su relación con la creatividad docente en profesores de nivel primario de Arequipa. Rev. Psicol Trujillo [revista en la internet]. 2014 [consultado 16 octubre de 2018];16(1):64-77. Disponible en: http://ucvvirtual.edu.pe/contenido_web/ Docs_Adic/20141022_REV_PSICO__2014-1.pdf

4. Karasek RA. Job demands, job decision latitude, and mental strain: implications for job redesign. AdmSci Q [revista en la internet]. 1979 [consultado 16 octubre en 2018]; 24: 285-308. Disponible en: https://www. jstor.org/stable/pdf/2392498.pdf?seq=1\#page_scan_tab_contents

5. Ceballos-Vásquez P, Rolo-González G, Hernández- Fernaud E, Díaz-Cabrera D, Paravic-Klijn T, BurgosMoreno M. Factores psicosociales y carga mental de trabajo: una realidad percibida por enfermera/os en unidades críticas. Rev. Latino-Am Enfermagem [revista en la internet]. 2015 [consultado 16 octubre de 2018]; 23(2): 315-322. Disponible en: http://www.scielo.br/pdf/rlae/v23n2/es_0104-1169-rlae-23-02-00315. pdf

6. Prochnow A, Magnago TSBS, Urbanetto JS, Beck CLC, Lima SBS, Greco PBT. Capacidad para el trabajo en enfermería: relación con demandas psicológicas y control sobre el trabajo. Rev. Latino-Am Enfermagem [revista en la internet]. 2013 [consultado 16 octubre de 2018]; 21(6):1298-1305. Disponible en: http:// www.scielo.br/pdf/rlae/v21n6/es_0104-1169-rlae-21-06-01298.pdf

7. Consejo Internacional de Enfermeras. Las enfermeras una fuerza para el cambio: un recurso vital para la salud. Ginebra: CIE; 2014. Disponible en: http://www.unq.edu.ar/advf/documentos/5370c85b69a41.pdf

8. Castillo I, Torres N, Ahumada A, Cárdenas K, Licona S. Estrés laboral en enfermería y factores asociados. Cartagena (Colombia) Salud Uninorte. Barranquilla (Col.) [revista en la internet]. 2014 [consultado 17 octubre de 2018]; 30 (1): 34-43. Disponible en: http://www.scielo.org.co/pdf/sun/v30n1/v30n1a05.pdf

9. Muñoz C, Rumie H, Torres G, Villarroel K. Impacto en la salud mental de la (del) enfermera(o) que otorga cuidados en situaciones estresantes. Cienc Enferm [revista en la internet]. 2015 [consultado 17 octubre de 2018]; XXI (1): 45-53. Disponible en: https://scielo.conicyt.cl/pdf/cienf/v21n1/art_05.pdf 
10. Carrillo-García C, Ríos-Rísquez M, Escudero-Fernández L, Martínez-Roche M. Factores de estrés laboral en el personal de enfermería del equipo volante según el Modelo de demanda-Control-Apoyo. Enferm Glob [revista en la internet]. 2018 [consultado 17 octubre 2018];17(2):304-314. Disponible en: http:// revistas.um.es/eglobal/article/view/277251/226341

11. Más Pons R, Escribá V. La versión castellana de la escala "The Nursing Stress Scale" proceso de adaptación transcultural. Rev. Esp Salud Pública [revista en la internet]. 1998 [consultado 16 octubre de 2018];72(6): 529-538. Disponible en: http://scielo.isciii.es/pdf/resp/v72n6/castellana.pdf

12. Garza R, Meléndez M, Castañeda H, Aguilera A, Acevedo G, Rangel S. Nivel de estrés en enfermeras que laboran en áreas de hospitalización. Desarrollo Cientif Enferm [revista en la internet]. 2011[consultado 17 octubre de 2018]; 19 (1): 15-19. Disponible en: http://www.index-f.com/dce/19pdf/19-015.pdf

13. Kogien M, Cedaro JJ. Emergencia Pública: impactos psicosociales en el dominio físico de la calidad de vida de profesionales de enfermería. Rev. Latino-Am. Enfermagem [revista en la internet]. 2014 [consultado 17 octubre de 2018]; 22(1). Disponible en: http://www.scielo.br/pdf/rlae/v22n1/es_0104-1169rlae-22-01-00051.pdf

14. Rodrigues VMCP, Ferreira ASS. Factores generadores de estrés en enfermeras en unidades de terapia intensiva. Rev. Latino-Am Enfermagem [revista en la internet]. 2011[consultado 17 octubre de 2018]; 19(4): [09 pantallas]. Disponible en: http://www.scielo.br/pdf/rlae/v19n4/es_23.pdf

15. Navarro J. Revisión de los estudios sobre profesionales de enfermería de las unidades de cuidados intensivos de España. Enferm Glob [revista en la internet]. 2012 [consultado 17 octubre de 2018]; 11(2):267289. Disponible en: http://scielo.isciii.es/pdf/eg/v11n26/revision1.pdf

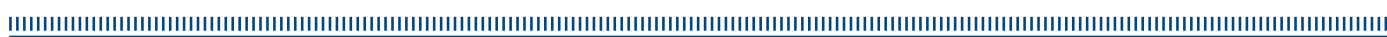

